\title{
Emergence of NDM-5-producing Escherichia coli sequence type 167 clone in Italy
}

\author{
Maria Giufrè ${ }^{\text {, Giulia Errico }}{ }^{a}$, Marisa Accogli ${ }^{a}$, Monica Monaco ${ }^{a}$, Laura Villa ${ }^{a}$, \\ Maria Antonietta Distasi ${ }^{b}$, Tito Del Gaudio ${ }^{b}$, Annalisa Pantosti ${ }^{a}$, Alessandra Carattoli ${ }^{a}$, \\ Marina Cerquetti ${ }^{a, *}$
}

a Department of Infectious Diseases, Istituto Superiore di Sanità, Viale Regina Elena 299, 00161 Rome, Italy

${ }^{\mathrm{b}}$ U.O. Clinical Pathology Unit, Lorenzo Bonomo Hospital, Andria, Italy

\section{A R T I C L E I N F O}

\section{Article history:}

Received 19 December 2017

Accepted 24 February 2018

\section{Keywords:}

Escherichia coli

ST167

NDM-5

Carbapenemase

Resistance plasmid

Multidrug resistance

\begin{abstract}
A B S T R A C T
The emergence of carbapenemase-producing Enterobacteriaceae (CPE) is a critical concern worldwide. In Italy, CPE isolates are very frequent, with the KPC enzyme types strongly predominant whereas the New Delhi metallo- $\beta$-lactamase (NDM) enzymes are extremely rare. Here we report the first detection of NDM-5-producing Escherichia coli sequence type 167 (ST167) isolates from two patients with urinary tract infection (Ec001 and Ec002 from urines), including one with colonisation (Ec003 from faeces) admitted to the same hospital 2 months apart in 2017. Minimum inhibitory concentrations (MICs) were determined by broth microdilution. The carbapenemase type was identified both by phenotypic and genotypic methods. Isolate genotypes were investigated by phylogenetic typing, multilocus sequence typing (MLST) and pulsed-field gel electrophoresis (PFGE). Next-generation sequencing (NGS) was used to obtain complete sequences of plasmids. The three $E$. coli isolates carried the $b l a_{\mathrm{NDM}-5}$ gene, shared the same resistance phenotype and belonged to ST167. By PFGE, isolates showed the same profile, suggesting that they were the same strain. NGS revealed that the $b l a_{\mathrm{NDM}-5}$ gene was located on a 99-kb multireplicon plasmid (designed pNDM-5-IT) with a peculiar scaffold constituted by four replicons of the IncF type (FIA, FIB and two copies of the FII replicon). pNDM-5-IT plasmid harboured multiple resistance and virulence determinants, including the arginine deaminase (ADI) cluster never found associated with plasmids before. Since NDM-5-producing E. coli ST167 has been regarded as a successful epidemic clone in China, the emergence of such a clone carrying a plasmid associated both with multiresistance and virulence could be a public-health threat.
\end{abstract}

(C) 2018 Elsevier B.V. and International Society of Chemotherapy. All rights reserved.

\section{Introduction}

The emergence and spread of carbapenemase-producing Enterobacteriaceae has become a worldwide public-health concern, although the geographical distribution of the different carbapenemase types varies considerably [1]. In Italy, the predominant carbapenemase is the KPC type disseminated especially among Klebsiella pneumoniae isolates; among the metallo- $\beta$-lactamase enzymes, VIM is found both in K. pneumoniae and Escherichia coli, whilst New Delhi metallo- $\beta$-lactamase (NDM) enzymes have rarely been detected [2-5]. In contrast, NDM-producing Enterobacteriaceae are abundant in the Indian subcontinent, the Balkans and Middle East regions, which act as a major reservoir [6]. Transferable plasmids carried by different clones of Enterobacteriaceae

\footnotetext{
* Corresponding author.

E-mail address: marina.cerquetti@iss.it (M. Cerquetti).
}

species mediate the geographical spread of NDM enzymes in nonendemic countries [7].

Among the Enterobacteriaceae, extraintestinal E. coli is the most prevalent pathogen causing both community- and healthcareassociated infections. Acquisition of NDM enzymes by E. coli isolates is a matter of concern since it would strongly limit the therapeutic options in a species that frequently carries multiresistance determinants. Knowledge regarding $b a_{\mathrm{NDM}}$ gene-harbouring clones and plasmids is important to understand the epidemiology of resistance and to control the spread of NDM-positive E. coli in the community and healthcare system and to monitor the dissemination of bla $a_{\mathrm{NDM}}$ genes among other enterobacterial species.

Here we report the emergence of an NDM-5-producing E. coli sequence type 167 (ST167) clone in Italy. The total plasmid content of the NDM-producing ST167 isolates and the complete se-

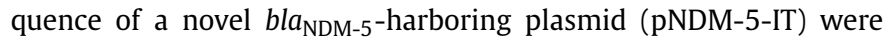
determined. 


\section{Materials and methods}

2.1. Strain identification, antimicrobial susceptibility testing and characterisation of resistance genes

Routine identification to species level and antimicrobial susceptibility testing were performed by automated methods $\left(\mathrm{VITEK}^{\circledR} 2\right.$; bioMérieux Italia S.p.A., Florence, Italy). Preliminary detection of NDM-type enzyme was carried out using the VERIGENE® Gramnegative Blood Culture (BC-GN) Test (Luminex, Hertogenbosch, The Netherlands). Antimicrobial susceptibility was confirmed by the reference broth microdilution method using the TREK Sensititre ${ }^{\mathrm{TM}}$ custom panel ITGNEGF (Thermo Fisher TREK Diagnostic Systems, Inc., Cleveland, $\mathrm{OH}$ ). The interpretative breakpoints were based on European Committee on Antimicrobial Susceptibility Testing (EUCAST) [8]. Phenotypic confirmatory testing for carbapenemase production was performed using agar tablet/disk diffusion method using the KPC/MBL and OXA-48 Confirm Kit (Rosco Diagnostica A/S, Taastrup, Denmark). Identification of carbapenemase-encoding genes (bla $a_{\mathrm{VIM}}, b a_{\mathrm{IMP}}, b l a_{\mathrm{NDM}}, b l a_{\mathrm{OXA}-48}$ and $\left.b l a_{\mathrm{KPC}}\right)$ and their variants was performed by PCR and sequencing [9]. Detection and sequencing of extended-spectrum $\beta$-lactamase (ESBL) and/or plasmid AmpC genes (bla $a_{\mathrm{CTX}-\mathrm{M}}, b l a_{\mathrm{SHV}}, b l a_{\mathrm{TEM}}$ and $b l a_{\mathrm{CMY}-2}$ ) was carried out as previously described [10].

\subsection{Genotyping}

NDM-producing $E$. coli isolates were assigned to one of the seven major E. coli phylogenetic groups (A, B1, B2, C, D, E and F) by the multiplex PCR-based method described by Clermont et al. [11]. Genetic relatedness among isolates was assessed by multilocus sequence typing (MLST) according to the MLST website (http: $/ / \mathrm{mlst}$.warwick.ac.uk/mlst/dbs/Ecoli) and by pulsed-field gel electrophoresis (PFGE) following previously reported procedures [12].

\subsection{Characterisation of plasmids}

Plasmids from isolates Ec001 and Ec002 were extracted using a PureLink ${ }^{\mathrm{TM}}$ HiPure Plasmid Filter Midiprep Kit (Invitrogen, Milan, Italy). Purified plasmid DNA was used for preparation of DNA paired-end libraries generated using the Nextera XT DNA Sample Preparation Kit (Illumina Inc., San Diego, CA) and was sequenced using an Illumina MiSeq instrument with $2 \times 300 \mathrm{PE}$ protocol (Illumina Inc.). De novo assembly of Illumina reads was performed using SPAdes 3.8 software through the ARIES public Galaxy server (https://w3.iss.it/site/aries/). Contigs were screened for plasmid and resistance gene content using PlasmidFinder and ResFinder tools, respectively, at the Center for Genomic Epidemiology (CGE) server (https://cge.cbs.dtu.dk/services/). Replicon alleles were assigned at the plasmid MLST site (https://pubmlst. org/plasmid/). The order and orientation of contigs with overlapping paired-ends was initially performed following the assembly of two reference plasmids, pCTXM15_EC8 ( KP789020.1) and FDAARGOS_434 unnamed plasmid 1 ( CP023871.1), identified in E. coli from China and Canada, respectively. The pNDM-5-IT complete sequence was confirmed by the PCR-based gap closure method and Sanger sequencing of the amplicons.

Circular plasmid sequences were annotated at the Rapid Annotation using Subsystem Technology (RAST) server (http://rast. nmpdr.org/).

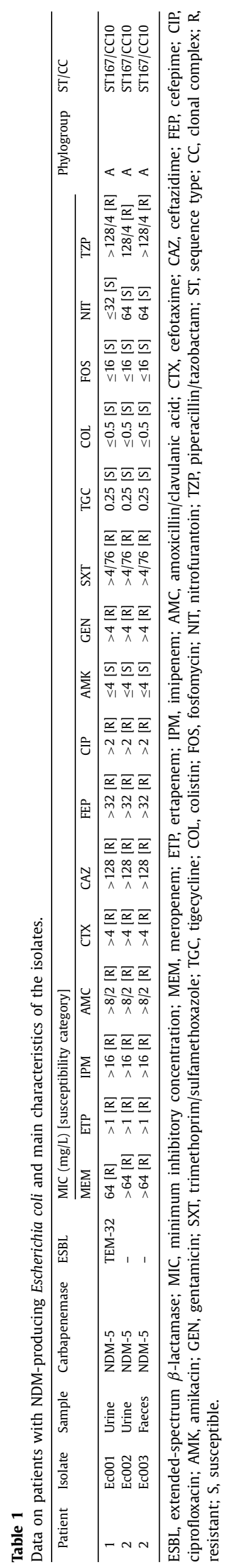


Table 2

Plasmid content of NDM-producing Escherichia coli isolates.

\begin{tabular}{|c|c|c|c|c|}
\hline Isolate & Plasmid & Size (bp) & Replicon content & Resistance genes \\
\hline \multirow[t]{7}{*}{ Ec001 } & pNDM-5-IT & 99476 & FII (allele 36) & $\begin{array}{l}\text { bla }_{\mathrm{NDM}-5}, \operatorname{aac}(3)-I I a, \\
\operatorname{aadA2}, \operatorname{mph} \text {, sul1, } \\
\text { tetA, dfrA12 }\end{array}$ \\
\hline & & & FII (allele 31) & \\
\hline & & & FIA (allele 4) & \\
\hline & & & FIB (allele 1) & \\
\hline & $\mathrm{pX} 1$ & 38611 & $\mathrm{X} 1$ & bla $a_{\mathrm{TEM}-32}$ \\
\hline & pMG2 & 5167 & Col156 & \\
\hline & pMG1 & 4773 & ColRNAI & \\
\hline \multirow[t]{6}{*}{ Ec002 } & pNDM-5-IT & 99476 & FII (allele 36) & $\begin{array}{l}\text { bla }_{\mathrm{NDM}-5}, \operatorname{aac}(3)-I I a, \\
\text { aadA2, mphA, sul1, } \\
\text { tetA, dfrA12 }\end{array}$ \\
\hline & & & FII (allele 31) & \\
\hline & & & FIA (allele 4) & \\
\hline & & & FIB (allele 1) & \\
\hline & pMG2 & 5167 & Col156 & \\
\hline & pMG1 & 4773 & ColRNAI & \\
\hline
\end{tabular}

\section{Results}

\subsection{Patients and NDM-producing Escherichia coli isolates}

The three NDM-producing E. coli isolates (Ec001, Ec002 and Ec003) analysed in this study were obtained from two patients aged 60-70 years with urinary tract infection (UTI) admitted to the same hospital (Hospital 'Lorenzo Bonomo', Andria, Puglia Region, Italy) 2 months apart in 2017. Patient 1 was admitted to the neurosurgery unit of the hospital, whilst Patient 2 presented to the outpatient clinic. Ec001 and Ec002 were recovered from the urine cultures of Patients 1 and 2, respectively, whilst Ec003 was detected in a rectal swab sample from Patient 2. No NDM-producing isolate was identified in the faeces of Patient 1 . Both patients were treated with fosfomycin although with a different therapeutic scheme (a single $3 \mathrm{~g}$ dose for Patient 1 and two $3 \mathrm{~g}$ doses $24 \mathrm{~h}$ apart for Patient 2) and both recovered. At a check-up 4 months later, Patient 2 was found to be negative for NDM-producing $E$. coli by urine culture, although faecal colonisation persisted. Neither patient reported a recent travel history to any NDM-endemic area. The three isolates (Ec001, Ec002 and Ec003) were sent to the Istituto Superiore di Sanità (Rome, Italy) for further phenotypic and genotypic characterisation.

\subsection{Antibiotic susceptibility profile and genotyping of Escherichia coli}

All three E. coli isolates shared the same resistance profile. As shown in Table 1, the isolates were resistant to meropenem, ertapenem, imipenem, amoxicillin/clavulanic acid, third- and/or fourth-generation cephalosporins, ciprofloxacin, gentamicin, trimethoprim/sulfamethoxazole and piperacillin/tazobactam but remained susceptible to nitrofurantoin, colistin, amikacin, fosfomycin and tigecycline. All three isolates were found to carry the bla $a_{\mathrm{NDM}-5}$ gene. Results of phenotypic tests were consistent with the type of carbapenemase produced. Ec001 also harboured the bla $a_{\mathrm{TEM}-32}$ ESBL gene, but the isolates from Patient 2 (Ec002 and Ec003) did not. No other carbapenemase/ESBL gene was detected. All three isolates belonged to phylogenetic group A, clonal complex 10 (CC10) and ST167. The isolates showed an identical profile by PFGE (data not shown).

\subsection{Plasmid content of Ec001 and Ec002}

Plasmid next-generation sequencing analysis was conducted on the two UTI isolates (Ec001 and Ec002) from Patients 1 and 2, respectively. PlasmidFinder performed on assembled contigs from Ec001 and Ec002 detected the same plasmid content in both isolates, with the exception of an X1 plasmid that was found in Ec001 isolate only (Table 2).

The plasmid carrying $b a_{\mathrm{NDM}-5}$ was designated pNDM-5-IT, belonged to the IncF family and also harboured dfrA12, aadA2, sul1, aac(3)-IIa, mphA and tetA(A) resistance genes. Plasmid pX1_1 carried only the $b l a_{\text {TEM-32 }}$ gene. Two different small plasmids (named pMG1 and pMG2) were also identified matching with the pIGMS32 (DQ298019) and pIGJC156 (NC_009781) sequences, respectively. These latter encoded the basic rolling circle replication controls and the MobA-L mobilisation systems.

\subsection{Features and characteristics of the PNDM-5-IT plasmid}

Within the pNDM-5-IT plasmid, the bla $a_{\mathrm{NDM}-5}$ gene was found in a complex integron, bracketed by two IS26, containing an ISCR1 element and a class 1 integron with the intI 1 gene truncated by one of the IS26 copies and the aadA2-dfrA12 resistance gene cassettes (Fig. 1).

pNDM-5-IT showed a peculiar scaffold constituted by four replicons of the IncF type (FIA, FIB and two copies of the FII replicon). Plasmid MLST assigned this plasmid to the FAB formula F36:F31:A4:B1. The FII allele 36 differs by one nucleotide from the FII allele 31 and is not functional since the replication protein repA gene was destroyed by the insertion of an IS1 element.

By BlastN, the best match with pNDM-5-IT (99\% nucleotide identity and $81 \%$ coverage) was observed with plasmid pCTXM15_EC8 identified in WCHEC13-8 E. coli strain ST3835 isolated in China [13]. pCTXM15_EC8 also showed FIA, FIB and two FII replicons, with the highly related $F A B$ formula $F 36: F 36: A 4: B 1$.

Comparative analysis between pNDM-5-IT and pCTXM15_EC8 showed that the scaffold of both plasmids was highly conserved, including the replicons, stabilisation, toxin-antitoxin systems (sopA, sopB, $c c d A, \operatorname{ccdB}, \operatorname{pemI}$, pemK) and putative virulence factors such as the arginine deaminase (ADI) cluster ( $\operatorname{arc} A$, $\operatorname{arc} B$, $\operatorname{arc} C$ and $\operatorname{arcD}$ genes) flanked by two inverted repeated IS66-IS1 elements but also the iron/manganese $A B C$ transporter and the aerobactin Iuc system (Fig. 1). The deleted FII replicon was trapped by the element constituted by two inverted repeated IS66-IS1 sequences carrying the ADI cluster. The Tra locus for conjugation was intact in pCTXM15_EC8 but was partial in pNDM-5-IT (only the traX and finO genes were detected), indicating that pNDM-5-IT is not able to promote self-conjugation. pCTXM15_EC8 and pNDM-5-IT differed for the resistance determinant content: pNDM-5-IT carried the dfrA12-aadA2-ISCR1-bla NDM-5 $_{2}$ complex integron and the macrolide (mphA-mphR) and gentamicin [aac(3)-IIa] resistance genes, whilst in the same plasmid region pCTXM15_EC8 showed the ISEcp1-bla aacA4, bla $a_{\mathrm{OXA}-1}$ and cat resistance genes. Plasmid pNDM-5-IT also matched (99\% nucleotide identity, 54\% coverage) with an NDM-5positive plasmid identified in an E. coli strain isolated in Canada (FDAARGOS_434).

pFDAARGOS_434 plasmid carried the FII and FIA replicons with a FAB formula F36:A4:B-. Comparison among plasmids showed that the region comprising the bla $a_{\mathrm{NDM}-5}$-containing integron and the $m p h A-m p h R$ genes was identical in pFDAARGOS_434 and pNDM-5-IT plasmids. The region encoding the FIB, the ABCaerobactin Iuc system identified in pCTX15_EC8 and pNDM-5IT was missing in pFDAARGOS_434, that showed, in this place, a different $A B C$ /iron transport system (Fig. 1). Finally, pFDAARGOS_434 lacked the ADI cluster with the second deleted copy of FII. 


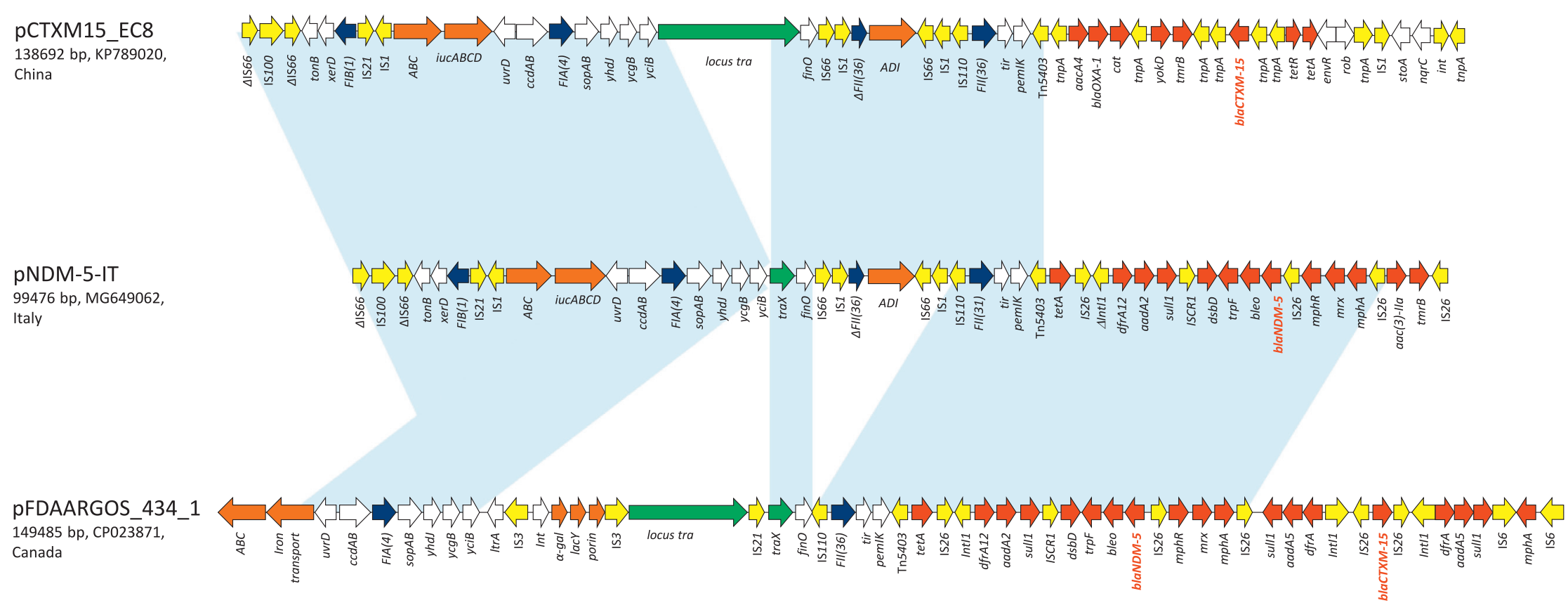

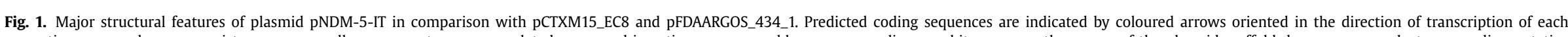

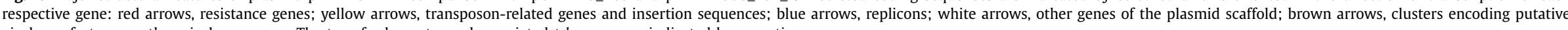
virulence factors or other virulence genes. The transfer locus tra and associated trb genes are indicated by a continuous green arrow. 


\section{Discussion}

Here we report on the first detection of E. coli ST167 isolates carrying an NDM-5 carbapenemase on a novel IncF plasmid in Italy. Enterobacteriaceae carrying NDM enzymes have rarely been observed in our country $[4,5,14,15]$. According to a recent survey on carbapenemase-producing Enterobacteriaceae in Europe (EuSCAPE), an irrelevant proportion of NDM-positive $K$. pneumoniae and/or $E$. coli isolates ( $0.5 \%$ and $0 \%$, respectively) was observed among Italian isolates [3].

The three NDM-5-producing isolates described in this study (i) displayed overlapping resistance phenotypes, (ii) belonged to the same ST167 clone, (iii) were identical by PFGE and (iv) harboured the same plasmid content, suggesting they were actually the same strain. Transmission of the strain from one patient to the other could be hypothesised but we were unable to document a direct epidemiological link. In the literature, ST167 was previously found associated with NDM-5 mainly in China where it was recognised as an epidemic clone of significant public-health concern carrying plasmids associated with antimicrobial resistance [16-18]. In Europe, the ST167 clone has been sporadically detected. In Italy, it was found to occur very rarely [10] or not at all [19]. As observed in China, the ST167 isolates detected in this study were highly resistant to several different classes of antimicrobial agents $[16,18]$. Accordingly, besides the bla $a_{\mathrm{NDM}-5}$ gene, plasmid pNDM-5IT harboured other resistance determinants conferring resistance to trimethoprim, sulfonamides, aminoglycosides, macrolides and tetracycline. However, it is noteworthy that the ST167 isolates remained susceptible to old antimicrobial agents such as nitrofurantoin and fosfomycin, the latter successfully used in the treatment of both of patients.

In Italy, the only other cases of infection due to an NDM-5carrying E. coli were found to be associated with ST405 [5]. The plasmid in the ST405 isolate belonged to the IncFII group but the scaffold was highly different from that identified herein in ST167. In fact, the pNDM-5-IT plasmid had a scaffold characterised by the double FII feature. Looking at the complete plasmid sequences available in GenBank, double alleles (either F36:F31 or F36:F36) were present in 17 plasmids, of which 15 also showed the FIA allele A4 (data not shown). Most of these plasmids were associated with the presence of the bla with the $b l a_{\mathrm{NDM}-5}$ gene. It is therefore plausible that either the bla $_{\mathrm{NDM}-5}$ or the bla $a_{\mathrm{CTX}-\mathrm{M}-15}$ gene had independent occasions to join this type of IncF plasmid that is very frequent in E. coli. Of note, in this study we noted the association of the double FII feature with the ADI cluster. The ADI pathway converting L-arginine into L-ornithine is the most widespread anaerobic route for arginine degradation contributing to survival of bacteria in acidic environments [20]. The ADI cluster is widely present in different bacterial species and genera, but it has not been previously described linked to a plasmid. This genetic determinant could be considered a plasmid-mediated virulence factor in addition to the aerobactin system for iron uptake also carried by the pNDM-5-IT plasmid.

In conclusion, an important feature of NDM-producers is their ability to spread rapidly both in healthcare and community settings [21]. Considering that E. coli is the leading cause of UTI, the occurrence of the NDM-5 enzyme in the ST167 clone that, although rare so far in Italy, is frequent in NDM-endemic regions such as China, is of concern. Moreover, the NDM-5-producing ST167 strain characterised herein was found to carry the bla $a_{\mathrm{NDM}-5}$ gene on a plasmid possessing both virulence and resistance features that in combination may facilitate its further spread. Overall, this study underlines the importance of surveillance and investigation of carbapenem-resistant clones and associated genes and plasmids.

\section{Sequence accession numbers}

Plasmid nucleotide sequences have been deposited in GenBank with the following accession nos.: MG649062 (pNDM-5-IT);

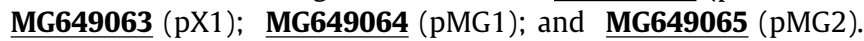

\section{Acknowledgment}

The authors are very grateful to Tonino Sofia for editorial assistance.

\section{Funding}

None.

\section{Competing interests}

None declared.

\section{Ethical approval}

Not required.

\section{References}

[1] Logan LK, Weinstein RA. The epidemiology of carbapenem-resistant Enterobacteriaceae: the impact and evolution of a global menace. J Infect Dis 2017;215:S28-36.

[2] Conte V, Monaco M, Giani T, D'Ancona F, Moro ML, Arena F, et al. Molecular epidemiology of KPC-producing Klebsiella pneumoniae from invasive infections in Italy: increasing diversity with predominance of the ST512 clade II sublineage. J Antimicrob Chemother 2016;71:3386-91.

[3] Grundmann H, Glasner C, Albiger B, Aanensen DM, Tomlinson CT, Andrasevic' AT, et al. Occurrence of carbapenemase-producing Klebsiella pneumoniae and Escherichia coli in the European survey of carbapenemase-producing Enterobacteriaceae (EuSCAPE): a prospective, multinational study. Lancet Infect Dis 2017;17:153-63.

[4] Gaibani P, Ambretti S, Berlingeri A, Cordovana M, Farruggia P, Panico M, et al. Outbreak of NDM-1-producing Enterobacteriaceae in northern Italy. July to August 2011. Euro Surveill 2011:16:20027.

[5] Bitar I, Piazza A, Gaiarsa S, Villa L, Pedroni P, Oliva E, et al. ST405 NDM-5 producing Escherichia coli in Northern Italy: the first two clinical cases. Clin Microbiol Infect 2017:23:489-90.

[6] Berrazeg M, Diene S, Medjahed L, Parola P, Drissi M, Raoult D, et al. New Delhi metallo-beta-lactamase around the world: an eReview using Google Maps. Euro Surveill 2014;19:20809.

[7] Khan AU, Maryam L, Zarrilli R. Structure, genetics and worldwide spread of New Delhi metallo- $\beta$-lactamase (NDM): a threat to public health. BMC Microbiol 2017;17:101

[8] European Committee on Antimicrobial Susceptibility Testing Breakpoint tables for interpretation of MICs and zone diameters: version 8.0, http://www.eucast.org/fileadmin/src/media/PDFs/EUCAST_files/ Breakpoint_tables/v_8.0_Breakpoint_Tables.pdf; 2018 [accessed 30.04.18]

[9] Accogli M, Giani T, Monaco M, Giufrè M, García-Fernández A, Conte V, et al. Emergence of Escherichia coli ST131 sub-clone H30 producing VIM-1 and KPC-3 carbapenemases. Italy. J Antimicrob Chemother 2014;69:2293-6.

[10] Giufrè M, Graziani C, Accogli M, Luzzi I, Busani L, Cerquetti M. Escherichia coli of human and avian origin: detection of clonal groups associated with fluoroquinolone and multidrug resistance in Italy. J Antimicrob Chemother 2012;67:860-7.

[11] Clermont O, Christenson JK, Denamur E, Gordon DM. The Clermont Escherichia coli phylo-typing method revisited: improvement of specificity and detection of new phylo-groups. Environ Microbiol Rep 2013;5:58-65.

[12] Cerquetti M, Giufrè M, García-Fernandez A, Accogli M, Fortini D, Luzzi I, et al. Ciprofloxacin-resistant, CTX-M-15-producing Escherichia coli ST131 clone in extraintestinal infections in Italy. Clin Microbiol Infect 2010;16:1555-8.

[13] Feng Y, Yang P, Xie Y, Wang X, McNally A, Zong Z. Escherichia coli of sequence type 3835 carrying bla NDM-1, bla CTX-M-15, bla CMY-42 and bla SHV-12. Sci Rep 2015;5:12275.

[14] Coppo E, Del Bono V, Ventura F, Camera M, Orengo G, Viscoli C, et al. Identification of a New Delhi metallo- $\beta$-lactamase-4 (NDM-4)-producing Escherichia coli in Italy. BMC Microbiol 2014;14:148.

[15] D’Andrea MM, Venturelli C, Giani T, Arena F, Conte V, Bresciani P, et al. Persistent carriage and infection by multidrug-resistant Escherichia coli ST405 producing NDM-1 carbapenemase: report on the first Italian cases. J Clin Microbiol 2011;49:2755-8

[16] Zhang X, Lou D, Xu Y, Shang Y, Li D, Huang X, et al. First identification of coexistence of blaNDM-1 and blaCMY-42 among Escherichia coli ST167 clinical isolates. BMC Microbiol 2013;13:282. 
[17] Yang P, Xie Y, Feng P, Zong Z. blaNDM-5 carried by an IncX3 plasmid in Escherichia coli sequence type 167. Antimicrob Agents Chemother 2014;58:7548-52.

[18] Zhu YQ Zhao JY, Xu C, Zhao H, Jia N, Li YN. Identification of an NDM-5-producing Escherichia coli sequence type 167 in a neonatal patient in China. Sci Rep 2016;6:29934.
[19] Giufrè M, Ricchizzi E, Accogli M, Barbanti F, Monaco M, Pimentel de Araujo F, et al. Colonization by multidrug-resistant organisms in long-term care facilities in Italy: a point-prevalence study. Clin Microbiol Infect 2017;23:961-7.

[20] Noens EE, Lolkema JS. Convergent evolution of the arginine deiminase pathway: the ArcD and ArcE arginine/ornithine exchangers. Microbiologyopen 2017;6:e00412.

[21] Dortet L, Poirel L, Nordmann P. Worldwide dissemination of the NDM-type carbapenemases in Gram-negative bacteria. Biomed Res Int 2014;2014:249856. 